\title{
Sympathetically mediated upper back pain after coronary artery bypass graft surgery
}

\author{
So-Young Kwon, Jin-Deok Joo, Jung Hyun Kim, and Jong-Tae Jeong \\ Department of Anesthesiology and Pain Medicine, St. Vincent Hospital, College of Medicine, The Catholic University of Korea, \\ Suwon, Korea
}

Coronary artery bypass graft (CABG) surgery to restore normal blood flow to the heart is one of the most common surgical procedures worldwide. However, many patients suffer from a frequent complication: post-CABG pain (PCP) syndrome. PCP is characterized by chronic, atypical pain that restricts physical activity in daily living and causes depression [1]. Accurate, early diagnosis and active treatment of PCP are therefore very important. The onset of PCP is related to various factors, including brachial plexus traction, scar pain, costochondral junction pain, and upper limb complex regional pain syndrome. This pain usually occurs in the left anterior chest wall, although PCP can also be located at the mid-line scar and the right chest wall [2]. However, we report a case of sympathetically mediated pain occurring in the left upper back area accompanied by autonomic dysfunction in the left arm after the CABG.

A 55 year-old male patient was diagnosed with coronary artery disease (CAD) when he visited the hospital with a myocardial infarction in March 2010, as he showed 60\% stenosis in the left anterior descending artery, $90 \%$ stenosis in the left circumflex artery, and $90 \%$ stenosis in the right coronary artery from coronary angiography. However, the diameter of the lesion was $\leq 1.5 \mathrm{~mm}$, making it difficult to perform percutaneous coronary intervention (PCI); thus, medical therapy was administered. In March 2012, while still on therapy, he was admitted to the hospital via the emergency room with the main complaints of sudden chest pain and radiating facial pain. One week later, he underwent CABG surgery using the left internal mammary artery and right saphenous vein. Ten days after the surgery, chest pain and facial radiating facial pain disappeared, but the patient was referred to our pain clinic still complaining of left upper back pain on the thoracic 1-4th dermatome as well as numbness with a cold sensation in the left arm. Intercostal nerve block of the pain sites on the left thoracic 2-3th and 3-4th level was performed with $0.375 \%$ ropivacaine. Despite the block, the patient continued to complain of extreme pain at the same site as well as numbness in the left arm and tingling sensation. At the pain clinic, the pain intensity in the left upper back area was assessed as having a visual analogue scale (VAS) of 9-10, while the left arm and hand showed slight weakness and pale skin on the ulnar side. The patient and his guardian were given information about PCP and its likelihood after CABG surgery. Left stellate ganglion block (SGB) was carried out seven times every other day using $0.375 \%$ ropivacaine $6 \mathrm{ml}$. For pain management, NSAIDs, tri-cyclic antidepressants (TCAs), and anticonvulsants were prescribed, and a single epidural block was conducted two times on the left T2 and T3 using a mixture of $0.09 \%$ ropivacaine $6 \mathrm{ml}$ and triamcinolone $10 \mathrm{mg}$. After the SGB and epidural block, the upper back pain improved to a VAS of 2-3, but the patient still complained of left hand numbness and weakness. After the patient's discharge, the pain clinic performed an epidural block twice on the left C7 and T1, as well as left SGB seven times over two weeks. While receiving treatments as an outpatient at the pain clinic, the left upper back pain resolved, and the symptoms of autonomic dysfunction such as numbness and

Corresponding author: Jin-Deok Joo, M.D., Ph.D., Department of Anesthesiology and Pain Medicine, St. Vincent Hospital, College of Medicine, The Catholic University of Korea, 93, Ji-dong, Paldal-gu, Suwon 442-723, Korea. Tel: 82-31-249-7214, Fax: 82-31-258-4212, E-mail: joojd@ catholic.ac.kr

() This is an open-access article distributed under the terms of the Creative Commons Attribution Non-Commercial License (http:// creativecommons.org/licenses/by-nc/3.0/), which permits unrestricted non-commercial use, distribution, and reproduction in any medium, provided the original work is properly cited. 
skin color change in the left arm noticeably improved without sequelae. The patient remained symptom-free and returned to work after a month.

Many studies have investigated the prevalence of PCP in connection to its onset mechanism. Eng and Wells [1] reported a PCP prevalence of $23 \%$, while another report put the figure at 56\% [3]. The mechanism of PCP is believed to be chest wall trauma-induced musculoskeletal nociceptive pain and neuropathic pain from secondary formation. During harvesting of the internal mammary artery, the anterior branch of the intercostal nerve is most likely to be injured, triggering stepwise central neuropathic pain [2]. In many cases, the characteristic profile of PCP accompanies sensation abnormalities with hypoesthesia, allodynia and hyperalgesia, which are mainly expressed from the thoracic 2-4th dermatome. In addition, autonomic dysfunction can accompany symptoms such as swelling, skin color change, and temperature change [4]. In our case, tingling and cold sensations were expressed in the left arm, the same side, in which sympathetic activity increased excessively in the same segmental site during the course of central sensitization of the peripheral nerve damage, causing neuropathic pain. In addition, there was simultaneous, sympathetically mediated radiating pain in the upper back area. It is important to differentiate PCP from anginal pain because, in many cases, patients themselves are already aware of them, and detailed pain profiling and history taking are important in making an accurate diagnosis. Other causes of chronic chest pain after CABG surgery are sternal wound infection, brachial plexus injury, and shoulder girdle pain. Despite the small number of studies on the treatment of PCP, there are some case reports of successful SGB [4]. Our case, involved a successful treatment experience with several SGBs and thoracic epidural block. It is thought that SGB and thoracic epidural block reduce the secretion of catecholamine and various pain substances [5].

In conclusion, peripheral nerve damage during harvesting of a blood vessel for CABG may cause autonomic dysfunction at the same segmental site, and sympathetically mediated radiating pain may occur in the left upper back area. Because PCP has high prevalence and can last from several months to several years, it is important to preemptively reduce the development of PCP at the initial stage.

\section{References}

1. Eng J, Wells FC. Morbidity following coronary artery revascularization with the internal mammary artery. Int J Cardiol 1991; 30: 55-9.

2. Mailis A, Chan J, Basinski A, Feindel C, Vanderlinden G, Taylor A, et al. Chest wall pain after aortocoronary bypass surgery using internal mammary artery graft: a new pain syndrome? Heart Lung 1989; 18: 553-8.

3. Eisenberg E, Pultorak Y, Pud D, Bar-El Y. Prevalence and characteristics of post coronary artery bypass graft surgery pain (PCP). Pain 2001; 92: 11-7.

4. Khan Mu, Ahmed I. Role of stellate ganglion block in post CABG sympathetically mediated chest pain. J Pak Med Assoc 2007; 57: 470-2.

5. Kirnö K, Friberg P, Grzegorczyk A, Milocco I, Ricksten SE, Lundin S. Thoracic epidural anesthesia during coronary artery bypass surgery: effects on cardiac sympathetic activity, myocardial blood flow and metabolism, and central hemodynamics. Anesth Analg 1994; 79: 107581. 